\title{
MODELAGEM E VALIDAÇÃO DO REGIME DE OPERAÇÃO DESCONTÍNUO EM UMA COLUNA DE DESTILAÇÃO HÍBRIDA
}

\author{
A. APIO $^{1}$ e N. P. G. SALAU \\ Universidade Federal de Santa Maria, Departamento de Engenharia Química \\ E-mail para contato: ${ }^{1}$ andressaapio@ hotmail.com
}

RESUMO - O comportamento dinâmico de uma coluna de destilação híbrida foi modelado, simulado e validado neste trabalho. O modelo estudado permitiu a obtenção da concentração do produto de topo e de fundo da coluna de destilação no regime descontínuo. Como correlação termodinâmica, foi utilizada a equação de van Laar para simular a concentração de etanol-água, demonstrando um resultado satisfatório comparado à linha de equilíbrio de mistura apresentada na literatura. As frações de etanol simuladas pelo software EMSO concordaram bem com os dados experimentais, apesar das aproximações na dinâmica da coluna de destilação. Além disso, os perfis de temperatura foram um fator chave para a validação dos modelos, produzindo um comportamento dinâmico esperado na concentração do produto detopo e de fundo na coluna de destilação. Finalmente, foi visto que o potencial da modelagem de processos na aplicação do controle de pureza do produto de topo e na otimização das condições operacionais do processo.

\section{INTRODUÇÃO}

O processo de separação industrial mais importante, segundo Gmehling (1998), é a destilação, no qual energia é utilizada para criar um sistema de fases líquido e vapor. A fim de colocar as fases líquida e vapor em contato, diferentes tipos de pratos ou recheios são utilizados, elevando a transferência de massa do processo. Para a destilação etanol-água, de acordo com a ANP (2012), deve ser assegurada uma composição de combustível em um valor desejável acima de $92,5^{\circ} \mathrm{INPM}(83,04 \mathrm{~mol} \%)$.

Para Mujtaba (2004), valores de propriedades de interesse (geralmente pressão, temperatura e concentração em ambas as fases) são indispensáveis para projetar e otimizar o processo de destilação.Assim, a destilação descontínua, também chamada de destilação batelada, ocorre com uma única carga inicial, a qual deve ser destilada até ser obtido o produto na quantidade e pureza desejada. Além disso, essa é uma operação unitária muito eficiente, que permite o fracionamento de uma mistura multicomponente em seus constituintes puros em uma única coluna. Warteret al. (2004), em seu trabalho, afirmam que devido a sua flexibilidade e baixo custo de investimento, a destilação descontínua está se tornando mais importante em indústrias que fabricam produtos de química fina e farmacêuticos. 
A modelagem fenomenológica, nesse quesito, possibilita conectar a composição do líquido e o número de pratos da coluna, considerando as propriedades termodinâmicas e físicas da operação unitária de destilação, além das características da mistura etanol-água.Para a simulaçãodo modelo foi utilizado o software EMSO, que possibilitou a solução do sistema dinâmico de equações algébrico-diferenciais resultante da modelagem da coluna de destilação através do método integrador DASSL.

\section{COLUNA DE DESTILAÇÃO HÍBRIDA}

A modelagem fenomenológica deste trabalho foi baseada no protótipo proposto por Mayer (2010), e está instalado no Laboratório de Engenharia de Processos, da Universidade Federal de Santa Maria. O equipamento geralmente funciona em regime de operação contínua, e é composto por um destilador em escala de bancada feito de vidro de borossilicato com uma altura efetiva de 190 cm, 4 cm de diâmetro interno e está dividida em três módulos. Dois módulos são preenchidos com anéis de Raschig (seção de retificação) e o terceiro módulo, uma coluna do tipo Vigreux e compõe a seção de esgotamento. As alturas dos módulos são, respectivamente, $56 \mathrm{~cm}, 78 \mathrm{~cm}$ e 56 $\mathrm{cm}$, do fundo para o topo.
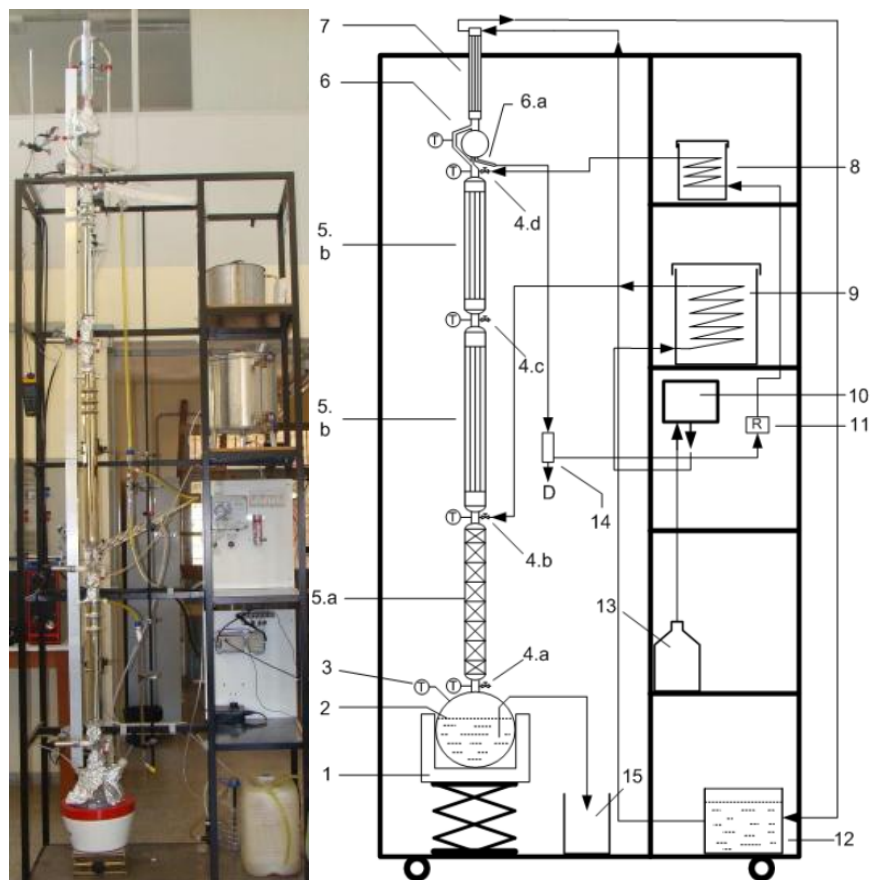

Figura 1 - Esquema do protótipo e imagem do equipamento instalado no Laboratório de Eng. de Processos

Legenda: 1 - Manta de aquecimento com suporte de altura ajustável; 2 - refervedor; 3 - sensor de temperatura; 4 - torneiras para retirada de amostra (4.a, b, c e d), entrada da alimentação (4.b) e retorno da vazão de refluxo (4.d); 5.a - coluna de destilação do tipo Vigreux; 5.b - coluna de destilação de recheios (anéis de Raschig); 6 - topo do destilador com condensador; 6.a. - registro de duas vias; 7 - condensador; 8 - tanque de pré-aquecimento com refluxo; 9 - tanque de pré-aquecimento da alimentação; 10 - bomba peristáltica da alimentação; 11 - medidor 
de refluxo; 12 - banho termostatizado para resfriamento e recirculação da água de condensação; 13 - tanque de armazenagem da alimentação; 14 - registro de controle de saída de destilado; 15 - tanque para coleta de produto de fundo.

A Figura 1 mostra um desenho esquemático do protótipo e uma imagem do equipamento utilizado.

\section{MODELAGEM DO PROCESSO DE DESTILAÇÃO DESCONTÍNUA}

A modelagem do modo de operação descontínuo, também conhecido como destilação em batelada, é baseada no modelo proposto por Domenech\&Enjalbert (1981). A alimentação é inserida na base da coluna, onde se localiza o destilador e, então, é aquecida para que ocorra o fluxo ascendente de vapor. À medida que o vapor formado no destilador avança ao longo dos pratos, fica mais rico no componente mais volátil (fase leve), até chegar ao topo e ser condensado. Após a condensação no topo da coluna, o produto pode retornar à coluna ou pode ser retirado como produto final. Quando o produto é retornado à coluna, é misturado novamente com o vapor. Isso permite uma transferência de massa adicional dos componentes da fase volátil para que a fase menos volátil e, como consequência, a corrente de vapor se torna mais rica do mais volátil, e a do líquido, do menos volátil. O retorno de líquido à coluna é chamado de refluxo, e a razão entre o destilado formado e o líquido que retorna à coluna é chamada de razão de refluxo (MUJTABA, 2004).

A fim de simplificar o modelo, Domenech\&Enjalbert (1981) propuseram as seguintes hipóteses a fim de reduzir o número de cálculos: coluna adiabática; acúmulo de vapor negligenciável; acúmulo de líquido constante ou zero; condensador total; pratos teóricos ou eficiência constante; pressão constante em cada prato; razão de refluxo e vazão de vapor mantidas constantes.

As equações do modelo representam o cálculo diferencial das composições no destilador, nos estágios intermediários e no condensador. As composições molares das frações líquida e vapor são $\mathrm{x}(j, i)$ e $\mathrm{y}(j, i)$, respectivamente, onde ' $j$ ' refere-se ao componente, e ' $i$ ' refere-se ao estágio de equilíbrio. Neste caso, o componente 1 é o etanol, e o 2 a água. Já os estágios de equilíbrio compreendem desde o destilador ( $12^{\circ}$ estágio) até o condensador ( $1^{\circ}$ estágio). $V$ é a vazão de vapor da mistura etanol-água que é a formada a partir do destilador, em $\mathrm{mol} / \mathrm{min} . M_{B}$ é o acúmulo molar no $12^{\circ}$ estágio (destilador), assim como $M_{S}$ é o acúmulo molar nos estágios intermediários, $M_{C}$ é o acúmulo molar no condensador e $M_{T}$ é o acúmulo molar no tanque. $\mathrm{x}_{\mathrm{D}}$ é a composição do destilado no tanque acumulador. Ainda, $R$ é o valor da razão de refluxo.As demais equações do modelo se resumem em equações diferenciais de balanço de massa e de energia, bem como a equação global da coluna, conforme a seguir:

O balanço para o condensador $(i=1)$ é dado pela equação 1 :

$$
\frac{d x(j, i)}{d t}=\frac{V}{M_{c}} \cdot(y(j, i+1)-x(j, i))
$$


O balanço para os demais pratos (de $i=2$ aNS-1) é dado pela equação 2:

$$
\frac{d x(j, i)}{d t}=\frac{V}{M_{S}} \cdot(y(j, i+1)-y(j, i))+\frac{V}{M_{S}} \cdot \frac{R}{(R+1)} \cdot(x(j, i-1)-x(j, i))
$$

O balanço para o refervedor $(i=N S)$ é dado pela equação 3:

$$
\frac{d x(j, i)}{d t}=\frac{V}{M_{B}} \cdot(x(j, i)-y(j, i))+\frac{V}{M_{S}} \cdot \frac{R}{(R+1)} \cdot(x(j, i-1)-x(j, i))
$$

O balanço global para o refervedoré dado pela equação 4:

$$
\frac{d M_{B}}{d t}=-D
$$

A remoção do destilado é dada pela equação 5:

$$
D=\frac{V}{(R+1)}
$$

O tanque acumulador é dado pela equação 6:

$$
\frac{d M_{T}}{d t}=D
$$

A composição do destilado no tanque acumulador é dada pela equação 7:

$$
\frac{d x D}{d t}=\frac{D}{M_{T}} \cdot\left(x_{N S}-x D\right)
$$

Por fim, o cálculo de equilíbrio entre as fases é feito mediante relação de equilíbrio representada na equação 8 , onde a fugacidade na fase líquida é estimada a partir do coeficiente de atividade $(\gamma(j, i))$, calculadapelas equações9 e 10 do modelo de van Laar, pela pressão de vapor saturado $\left(P_{S A T}(j, i)\right)$ e pela pressão do sistema $(P)$. Considera-se a fase vapor como ideal $\left(\phi^{\text {Vapor }}(j, i)=1\right)$.

$$
\begin{aligned}
& y(j, i)=x(j . i) \cdot \frac{P_{S A T}(j, i)}{P} \\
& \ln \gamma_{1}=A_{12}\left(\frac{A_{21} x_{2}}{A_{12} x_{1}+A_{21} x_{2}}\right)^{2} \\
& \ln \gamma_{2}=A_{21}\left(\frac{A_{12} x_{1}}{A_{12} x_{1}+A_{21} x_{2}}\right)^{2}
\end{aligned}
$$


Onde os parâmetros $\mathrm{A}_{12}$ e $\mathrm{A}_{21}$ do modelo de van Laar para a mistura binária etanol-água são dados por Perry (2009): $A_{12}=1.6798 e A_{21}=0.9227$.

\subsection{Condições iniciais do processo para a simulação do modelo}

As características da coluna de destilação híbrida no modo de operação descontínuo, tal qual o número de estágios, razão de refluxo, composição inicial e quantidade de energia fornecida ao destilador foram determinadas baseadas nas propriedades previamente calculadas por Mayer et al. (2012) para o modo de operação contínuo. No entanto, o acúmulo relativo aos diferentes pratos foi definido em face do processo de operação experimental da coluna de destilação. Para simular o modelo, todas as propriedades necessárias são mostradas na Tabela 1.

Tabela 1 - Propriedades do modelo contínuo para o processo de destilação.

\begin{tabular}{|c|c|c|}
\hline Símbolo & Propriedade & Valor \\
\hline$V_{R}$ & Fluxo molar de vapor & variável \\
\hline$D$ & Remoção molar de destilado & variável \\
\hline$R$ & Razão de refluxo & 6 \\
\hline$M_{D}$ & Acúmulo no destilador & $540 \mathrm{mols}$ \\
\hline$M_{S}$ & Acúmulo nos pratos & $540 \mathrm{mols}$ \\
\hline$M_{B}$ & Acúmulo no refervedor & $32000 \mathrm{mols}$ \\
\hline$M_{T}$ & Acúmulo no tanque & variável \\
\hline$x D$ & Composição de destilado no acumulador & variável \\
\hline$x(1,12)$ & Composição inicial & $0,032 \mathrm{~mol}$ \\
\hline$N S$ & Número de estágios & 12 \\
\hline$Q$ & Energia fornecida ao destilador & $990 \mathrm{~W}$ \\
\hline
\end{tabular}

\section{SIMULAÇÃO DO PROCESSO DE DESTILAÇÃO DESCONTÍNUO}

O modelo descrito no item 3 foi simulado no software EMSO utilizando as condições dadas pelo item 3.1. O resultado da simulação do modelo fenomenológico descrito no trabalho é mostrado na Figura 2. Conforme se pode observar, o resultado da simulação tem comportamento satisfatório, pois se têm duas etapas: start-up e produção de destilado. Além disso, a composição da massa líquida é modificada com o tempo, uma vez que o vapor formado é sempre mais rico no componente mais volátil. Quando a composição de etanol no topo atinge 5 minutos, o produto puro é obtido como destilado durante 30 minutos e, posteriormente, conforme o etanol na alimentação é extinto, a concentração de etanol no primeiro prato decresce. 


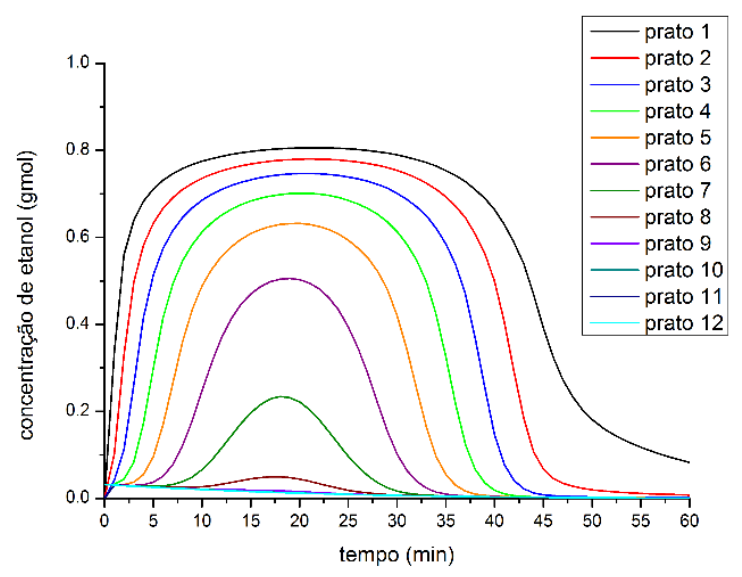

Figura 2-Perfis de composição simulados para o modelo de destilação descontínua.

\section{VALIDAÇÃO DA DESTILAÇÃO DESCONTÍNUA}

A coluna de destilação híbrida descrita no item 2 foi operada no modo de operação descontínuo, ou seja, somente com uma única carga inicial em uma operação intrinsecamente transiente. Durante a operação experimental, os perfis de temperatura da fase líquida no primeiro e último prato foram mensurados. A fim de checar os resultados gerados por simulação computacional, a validação experimental da simulação do processo foi conduzida, comparando os resultados obtidos através dos dados coletados na operação da coluna de destilação híbrida. Para esse propósito, amostras experimentais do produto foram coletadas e mensuradas através do densímetro, obtendo-se a composição molar, conforme pode ser observado na Tabela 2.

Tabela 2 - Composições experimentais e simuladas de etanol na destilação descontínua.

\begin{tabular}{|c|c|c|c|c|}
\hline Tempo (min) & $\begin{array}{c}\text { Concentração de } \\
\text { topo } \\
\text { experimental } \\
\text { (gmol) }\end{array}$ & $\begin{array}{c}\text { Concentração } \\
\text { de topo } \\
\text { simulada (gmol) }\end{array}$ & $\begin{array}{c}\text { Concentração } \\
\text { de fundo } \\
\text { experimental } \\
\text { (gmol) }\end{array}$ & $\begin{array}{c}\text { Concentração } \\
\text { de fundo } \\
\text { simulada } \\
\text { (gmol) }\end{array}$ \\
\hline 12 & 0,8255 & 0,7976 & - & 0,0158 \\
\hline 13 & 0,8140 & 0,8001 & - & 0,0150 \\
\hline 14 & 0,7923 & 0,8022 & - & 0,0142 \\
\hline 15 & 0,8031 & 0,8038 & - & 0,0135 \\
\hline 16 & 0,8031 & 0,8050 & - & 0,0127 \\
\hline 18 & 0,8035 & 0,8061 & - & 0,0114 \\
\hline 20 & 0,7930 & 0,8057 & - & 0,0102 \\
\hline 22 & 0,7942 & 0,8037 & - & 0,0091 \\
\hline 24 & 0,7911 & 0,7997 & - & 0,0080 \\
\hline 26 & 0,7803 & 0,7937 & - & 0,0071 \\
\hline
\end{tabular}




\begin{tabular}{|l|l|l|l|l|}
\hline 27 & 0,7835 & 0,7896 & - & 0,0067 \\
\hline 30 & 0,7742 & 0,7720 & - & 0,0056 \\
\hline 32 & 0,7696 & 0,7537 & 0,0084 & 0,0049 \\
\hline
\end{tabular}

A comparação entre os dados simulados através do modelo matemático e os dados experimentais dos pratos de topo (1) e de fundo (12) demonstrou que o modelo proposto prediz adequadamente o desempenho da coluna de destilação em questão.
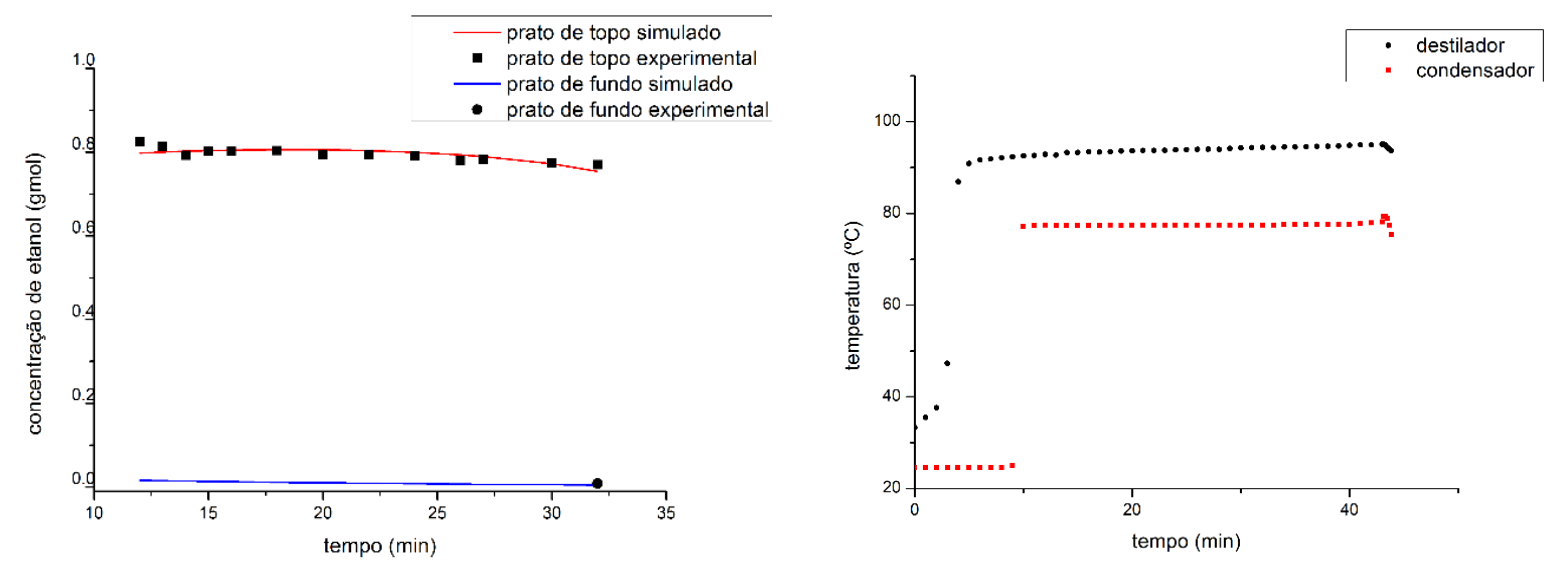

Figura 3 - a) Composições experimentais e simuladas de etanol na destilação descontínua. b) Perfis de temperatura para a coluna de destilação.

A Figura 3a mostra que os pequenos desvios na composição do produto analisado podem se dar devido às dificuldades de coletar o destilado, considerando o tempo de amostragem de apenas um ou dois minutos. Considerando que na operação descontínua a alimentação é introduzida em batelada, somente é possível analisar a composição de fundo no fim do processo. Portanto, a composição da última amostra resultou em um valor próximo ao comportamento do último prato simulado. Já a Figura 3bexibe os perfis de temperatura para condensador e o destilador. No tempo de cinco minutos, a temperatura no condensador subiu drasticamente até $77,404^{\circ} \mathrm{C}$, enquanto que no destilador a temperatura subiu até $95,5^{\circ} \mathrm{C}$. Os perfis de temperatura concordam com os perfis de composição experimentais ao longo da coluna. No condensador, a temperatura se manteve em $77,404^{\circ} \mathrm{C}$ por 30 min. Devido à queda de pressão, mantida a cerca de $0,95 \mathrm{~atm}$, a temperatura de $77,404^{\mathrm{a}} \mathrm{C}$ indica que etanol puro foi obtido. Após esse tempo, a temperatura decresce, indicando o término da destilação descontínua.

\section{CONCLUSÃO}

Uma das atividades mais relevantes no processo de destilação atualmente está relacionada à modelagem, para estudos de controle do sistema, análise da planta ou otimização. Nesse trabalho, 
foi possível validar o modelo para destilação descontínua para a coluna de destilação em bancada em questão, obtendo-se resultados satisfatórios na simulação quando comparados aos dados experimentais da planta. Além disso, o perfil de temperatura obtido experimentalmente na operação da coluna assegurou a pureza do destilado, além de ratificar o tempo de operação em batelada, conforme obtido através da simulação do modelo. Para a coluna de destilação desse trabalho, portanto, o modelo do processo estudado pode ser útil para o controle da pureza do produto, a fim determinar ao seudesempenho baseado em certas hipóteses, além de poder obter previsão das especificações mais adequadas para operar a coluna.

\section{REFERÊNCIAS}

ANP - Agência Nacional do Petróleo, Gás Natural e Biocombustíveis. (2005). Resolução ANP $\mathrm{n}^{\circ}$ 56, de 06 de dezembro de 2005. Disponível em <http://www.anp.gov.br>. AccessadoemJunho de 2012.

DOMENECH, S.; ENJALBERT, M. Program for Simulating Batch Rectification as a Unit Operation.Computers and Chemical Engineering.Vol. 5, No. 3, pp. 181-184, 1981.

GMEHLING, J.; MOLlMANN, C. Synthesis of Distillation Processes Using Thermodynamic Models and the Dortmund Data Bank.Ind. Eng. Chem. Res. 37, 3112-3123, 1998.

MAYER, F. D. Desenvolvimento da tecnologia de destilação apropriada à produção de álcool combustível em pequena escala. Dissertação de Mestrado PPGEPRO, Universidade Federal de Santa Maria, Santa Maria, 2010.

MUJTABA, I. M. Batch Distillation: Design and Operation. Series on Chemical Engineering Volume 3.Imperial College Press, London, 2004.

PERRY, R. H. Chemical Engineering Handbook. 7th edition. McGraw-Hill, New York, 1999.

WARTER, M.; DEMICOLI, D.; STICHMAIR, J. Operation of a batch distillation column with a middle vessel: experimental results for the operation of zeotropic and azeotropic mixtures. Chemical Engineering and Processing 43, p. 263-272, 2004. 